\title{
Uso da Cataia para a Produção de Aguardente Composta
}

Luciele Milani Zem

\begin{tabular}{|c|c|}
\hline & E-mail: luzem@uol.com.br \\
\hline $\begin{array}{l}\text { Katia Christina Zuffellato- } \\
\text { Ribas }\end{array}$ & $\begin{array}{l}\text { Departamento de Botânica. Universidade } \\
\text { Federal do Paraná, Curitiba, PR, Brasil. } \\
\text { E-mail: kazu@ufpr.br }\end{array}$ \\
\hline Maria Izabel Radomski & $\begin{array}{l}\text { Embrapa Florestas, Colombo, PR, Brasil. } \\
\text { E-mail: maria.radomski@embrapa.br }\end{array}$ \\
\hline Henrique Soares Koehler & $\begin{array}{l}\text { Universidade Estadual do Rio Grande do Sul, } \\
\text { Unidade Novo Hamburgo. Novo Hamburgo, } \\
\text { RS, Brasil. }\end{array}$ \\
\hline & E-mail: koehler@ufpr.br \\
\hline Agenc & $\begin{array}{l}\text { Laboratório de Tecnologia e Produtos Agríco- } \\
\text { las. Universidade Federal do Paraná, Curitiba, } \\
\text { PR, Brasil. }\end{array}$ \\
\hline
\end{tabular}
ba, PR, Brasil.

E-mail: luzem@uol.com.br

Departamento de Fitotecnia e Fitossanitarismo. Universidade Federal do Paraná, Curiti(n) 
preparadas em quatro tempos de infusão de trinta, sessenta, noventa e cento e vinte dias. No segundo experimento foram utilizadas dois $\mathrm{g} \mathrm{L}^{-1}$ de folhas secas de Drimys brasiliensis nos mesmos tempos de infusão, as quais foram adicionadas em cachaça não enveIhecida com $47,5 \%$ e $39,0 \%$ de álcool e aguardente de cana não envelhecida com $39,0 \%$ de álcool. Para a análise sensorial, uma equipe julgadora definiu as amostras a serem utilizadas, num total de seis por experimento e os testes de preferência foram realizados em escala Hedônica. Conclui-se que a cachaça com quatro $\mathrm{g} \mathrm{L}^{-1}$ de folhas de Pimenta pseudocaryophyllus foi a mais aceita pelos consumidores.

Palavras-chave: Análise Sensorial. Cachaça. Cataia. Folhas. Infusão.

\section{Abstract \\ Use of Cataia for the Production of Compo- site "Aguardente"}

Drimys brasiliensis and Pimenta pseudocaryophyllus, although being different species, share the same common name cataia and their leaves are flavouring of "cachaça". Therefore this study was to evaluate the use of the leaves of both species for the production of brandy made by infusion. Dry leaves of Pimenta pseudocaryophyllus, green and dry leaves of Drimys brasiliensis were used in the first experiment. Each sample was weighted in one, two and four $\mathrm{g} \mathrm{L}^{-1}$ and placed in infusion into glass bottles during four infusion periods of thirty, sixty, ninety and on hundred and twenty days. In the second experiment, two $\mathrm{g} \mathrm{L}^{-1}$ of Drimys brasiliensis dry leaves were used 
during the same infusion periods, placed into not aged "cachaça" with $47.5 \%$ and $39.0 \%$ alcohol content and into not aged sugar cane "aguardente" with $39.0 \%$ alcohol content. For the sensory analysis used six samples for each experiment and preference tests were performed asking the guests to express appreciation of drinks on an Hedonic scale. It follows that "Cachaça" with four $\mathrm{g} \mathrm{L}^{-1}$ of Pimenta pseudocaryophyllus was the most accepted by consumers.

Keywords: Sensory analysis. Cachaça. Cataia. Leaves. Infusion.

\section{Introdução}

Drimys brasiliensis Miers é uma espécie arbórea nativa da Mata Atlântica, encontrada entre as Florestas Tropicais mais ameaçadas e biodiversas do planeta (TRINTA; SANTOS, 1997). Pimenta pseudocaryophyllus (Gomes) Landrum é uma espécie arbórea comum no Sul do Brasil, sobretudo na Floresta Ombrófila, ocorrendo na caatinga da Bahia e na mata Atlântica do alto da Serra do Mar desde o Estado de São Paulo até o Estado de Santa Catarina (GlRARD, 2005).

Ambas as espécies são comumente conhecidas como cataia, apresentando uma importância econômica, uma vez que suas folhas são artesanalmente adicionadas à cachaça ou licor, pela população rural que habita nas proximidades dessa espécie (ZEM, 2014). A cachaça é considerada a segunda bebida alcóolica mais consumida no Brasil e vem conquistando mercados em razão dos esforços do setor produtivo (SORATTO; VARVAKIS; HORII, 2007). Já a aguardente de cana é a terceira bebida alcóolica mais consumida no mundo e a primeira no Brasil (BARBOZA et al., 2010). O impacto da Rev. Elet. Cient. UERGS, v. 3, n. 2, p. 266-285, 2017 
produção de cachaça e a ampliação do mercado vêm incentivando melhorias, controles mais rígidos e estudos mais detalhados, quer sobre a produção, qualidade química e atributos sensoriais da bebida (RECHE; FRANCO, 2009).

A aguardente e a cachaça são apreciadas devido ao seu sabor e aroma característicos, os quais são decorrentes dos processos de fermentação, destilação e envelhecimento da cana de açúcar. Impulsionado pela necessidade de conquista do mercado externo, existe um esforço do setor produtivo e dos laboratórios de pesquisa para a melhoria da qualidade da cachaça. A descrição qualitativa e quantitativa dos compostos químicos presentes tem recebido constante atenção por parte de diversos centros de pesquisa. Porém, a caracterização da cachaça, sobre o ponto de vista químico, não é suficiente, necessitando ser complementada pelo conhecimento dos atributos sensoriais da bebida (ODELLO et al., 2009).

O aprimoramento da qualidade e padronização da cachaça e aguardente é essencial para que a bebida atenda aos padrões internacionais, além de proporcionar maior aceitação no mercado interno, visto que um consumidor percebe quando duas bebidas são distintas simplesmente pela prova, exigindo assim bebida de boa qualidade (MIRANDA et al., 2007).

Diante do exposto, verifica-se a importância do estudo da acidez na qualidade da bebida, devido exercer influência nos resultados finais de sabor e aroma (PARAZZI et al., 2008). Quanto menor a acidez melhor são as características sensoriais da aguardente e maior aceitação pelos consumidores (MIRANDA et al., 2008). No entanto, a acidez não é uma característica exclusiva para a qualidade da cachaça (MARINHO et al., 2009).

Além da acidez, a cataia altera a cor, sabor e aroma. Quando utilizadas folhas de Pimenta pseudocaryophyllus, a cor da cacha- 
ça torna-se semelhante ao uísque e o sabor lembra madeira com leve toque de cravo. Já quando se utilizam folhas de Drimys brasiliensis, a cor lembra uísque, porém mais claro e o sabor é característico de pimenta. Diante das dificuldades em se obter informações sobre a produção da cachaça de cataia viu-se a necessidade do presente estudo, uma vez que se trata de uma bebida tradicionalmente conhecida no litoral do Paraná.

Sendo assim, o presente trabalho objetivou avaliar o uso de folhas de Drimys brasiliensis e Pimenta pseudocaryophyllos para a produção de aguardente composta por infusão, caracterizando o efeito de fatores como proporção (vegetal: bebida) e tempo de infusão sobre o produto final, somado aos atributos sensoriais da cachaça.

\section{Materiais e Métodos}

Este trabalho foi realizado em duas etapas ou experimentos distintos, cada uma avaliando diferentes variáveis do processo. Assim, no primeiro experimento foram estudados o material vegetal (a espécie, o processamento das folhas e a quantidade empregada) e o tempo de infusão (extração). No segundo experimento foi avaliado o solvente, neste caso o tipo de bebida e a graduação alcoólica, bem como o tempo de infusão ou contato entre folhas e a bebida alcoólica. O experimento teve início em junho de 2013 e finalizou em novembro do mesmo ano. 


\section{Avaliação do material e do tempo de infusão}

O primeiro experimento utilizou-se de folhas de cataia de duas espécies, Pimenta pseudocaryophyllus e Drimys brasiliensis e, dois processamentos, folhas verdes e secas. Para tanto foram colhidas na Fazenda Experimental da Embrapa Florestas localizada em Colombo (PR), folhas sadias de Drimys brasiliensis, as quais foram lavadas (tratamento folhas verdes) e uma parte colocada para secar em estufa, temperatura a $30^{\circ} \mathrm{C}$ (tratamento folhas secas). $\mathrm{E}$ foram usadas folhas secas de Pimenta pseudocaryophyllus, as quais foram cedidas pelas Produtoras de Cataia de Barra do Ararapira, produzida e embalada na área de proteção ambiental do Município de Guaraqueçaba, Litoral Norte do Estado do Paraná. Isto resultou em três diferentes tratamentos quanto ao material, como apresentado na tabela 1.

Tabela 1- Descrição do primeiro experimento usando folhas de Drimys brasiliensis Miers e Pimenta pseudocaryophyllus (Gomes) Landrum, Curitiba (PR), 2013.

\begin{tabular}{cl}
\hline Tratamento & \multicolumn{1}{c}{ Descrição } \\
\hline M1 & Folha seca litoral Pimenta peudocaryophyllus \\
M2 & Folha seca Colombo Drimys brasiliensis \\
M3 & Folha verde Colombo Drimys brasiliensis \\
Q1 & um g L-1 \\
Q2 & dois g L \\
Q3 & quatro g L \\
T1 & 30 dias \\
T2 & 60 dias \\
T3 & 90 dias \\
T4 & 120 dias \\
\end{tabular}

Além do fator material vegetal neste ensaio foi avaliada a quantidade de folhas posta em infusão, sendo usadas três doses ou quantidades, como descrito na Tabela 1. Cada tipo de material foi pesado nas quantidades estabelecidas e as folhas colocadas em garrafas de vidro com capacidade de setecentos $\mathrm{ml}$. As folhas foram 
então cobertas com cachaça não envelhecida com 39,0\% de álcool e colocadas em sala escura, à temperatura ambiente.

Foram coletadas amostras em diferentes tempos (Tabela 1), sendo que cada amostra coletada representava uma garrafa e no momento da coleta as folhas eram removidas da solução, interrompendo o processo de extração. Assim, combinando os fatores de variação, obteve-se trinte e seis tratamentos, os quais foram montados em duplicata (duas repetições) totalizando setenta e duas garrafas.

\section{Avaliação do solvente usado e do tempo de infusão}

Para o segundo experimento foi padronizado o material (foIhas de Drimys brasiliensis coletadas em Colombo (PR), secas em estufa a temperatura de $30^{\circ} \mathrm{C}$ ) e quantidade de folhas para infusão (concentração de dois $\mathrm{g} \mathrm{L}^{-1}$ ). As folhas foram colocadas em infusão usando garrafas de vidro com capacidade de setecentos $\mathrm{ml}$, preenchidas com cada uma das soluções testadas e colocadas em sala escura, a temperatura ambiente. Foram empregadas três diferentes soluções ou solventes e preparadas garrafas para a tomada de amostra nos quatro tempos previstos, como descrito na Tabela 2, obtendo-se doze tratamentos, em duplicata, ou vinte e quatro garrafas.

Tabela 2 - Descrição do segundo experimento usando folhas de Drimys brasiliensis Miers, Curitiba (PR), 2013.

\begin{tabular}{cl}
\hline Tratamento & \multicolumn{1}{c}{ Descrição } \\
\hline C1 & Cachaça não envelhecida / 47,5\% de álcool \\
C2 & Cachaça não envelhecida / 39,0\% de álcool \\
C3 & Aguardente de cana não envelhecida / 39,0\% de álcool \\
T1 & 30 dias \\
T2 & 60 dias \\
T3 & 90 dias \\
T4 & 120 dias \\
\hline
\end{tabular}




\section{Análises realizadas}

\section{Determinação da cor}

Foram coletadas alíquotas do produto de cada garrafa a cada 30 dias para a leitura de cor em espectrofotômetro e leitura de absorbância na faixa de $430 \mathrm{~nm}$. No momento da coleta destas alíquotas, as garrafas foram agitadas manualmente.

\section{Determinação da acidez}

Ao final dos 120 dias foram coletados $25 \mathrm{ml}$ de cada amostra e transferida para um béquer de $500 \mathrm{ml}$ contendo $200 \mathrm{ml}$ de água destilada. Em seguida a amostra foi titulada com uma solução de hidróxido de sódio $0,05 \mathrm{~N}$ até obter-se um pH de 8,2, com o uso de

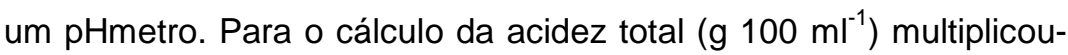
se o equivalente grama do ácido acético (sessenta) pelo volume da solução de hidróxido de sódio gasto na titulação $(\mathrm{ml})$ e pela normalidade da solução de hidróxido de sódio $(0,05)$; em seguida o resultado foi dividido pelo volume da amostra $(\mathrm{ml})$ multiplicado por 10 (BRASIL, 1986).

\section{Análises sensoriais}

Para a realização deste estudo experimental foi enviado ao comitê de ética em pesquisa (CEP/SD) da Universidade Federal do Paraná (UFPR), na qual obteve-se parecer favorável, certificado pelo processo ${ }^{\circ}$ CAAE 13421613.9.0000.0102. As normas de conduta para ingestão de bebida alcoólica no ensino, pesquisa e extensão do CEP/SD, foram rigorosamente seguidas sob coordenação da responsável pela pesquisa. 
Para a realização das análises sensoriais uma equipe julgadora definiu quais as amostras seriam utilizadas para a análise devido ao grande número de amostras. Definiu-se utilizar seis amostras para o primeiro experimento e seis para o segundo, conforme a Tabela 3 .

Os testes sensoriais afetivos foram conduzidos pela equipe do laboratório de Tecnologia de Produtos Agrícolas, Departamento de Solos e Engenharia Agrícola, UFPR, Curitiba (PR), divididos em dois dias, sendo um para o primeiro experimento e o outro dia para o segundo experimento. Foram colocados em edital, na Universidade Federal do Paraná convites com as características da pesquisa, recomendações e cuidados de segurança (requisitos) aos participantes, junto com datas e local para as avaliações.

Estas avaliações foram conduzidas individualmente e as amostras foram servidas a temperatura ambiente, em taças plásticas descartáveis, codificadas com três dígitos, em temperatura ambiente. Junto com as amostras foi servida água mineral a fim de eliminar o sabor residual entre os testes de uma amostra e outra.

Antes de cada julgamento foi feita uma preleção para o julgador sobre os objetivos do trabalho e os cuidados necessários no consumo de bebidas alcoólicas, em seguida cada convidado assinou um termo de consentimento livre e esclarecido, no qual informava todos os procedimentos e requisitos da análise sensorial. Posteriormente o julgador pode avaliar as amostras.

Diante do volume de tratamentos realizados, cada convidado recebeu apenas seis amostras, ou seja, avaliou apenas seis tratamentos do total realizado, no qual cada amostra tinha apenas cinco $\mathrm{ml}$ de bebida. Foi orientado aos mesmos para não consumirem todo o volume. Todas as amostras, num total de seis, foram apresentadas de uma única vez a fim de poder ser realizada uma análise 
comparativa. As fichas foram preenchidas de acordo com a preferência dos atributos em questão.

Tabela 3 - Amostras escolhidas para a análise sensorial de infusão de cachaça com Drimys brasiliensis Miers e Pimenta pseudocaryophyllus (Gomes) Landrum do primeiro e segundo experimentos, Curitiba (PR), 2013.

\begin{tabular}{|c|c|}
\hline Amostras & Descrição \\
\hline $\begin{array}{l}\text { M1Q1T4 } \\
\text { M1Q2T4 } \\
\text { M1Q3T4 } \\
\text { M2Q1T4 } \\
\text { M2Q2T4 } \\
\text { M2Q3T4 }\end{array}$ & 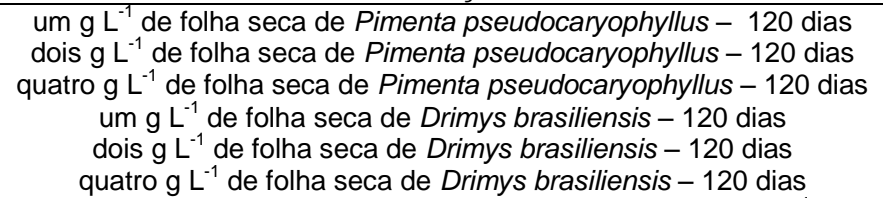 \\
\hline M4C & $\begin{array}{l}\text { Cachaça não envelhecida, com } 47,5 \% \text { de álcool com dois } \mathrm{g} \mathrm{L}^{-1} \text { de } \\
\text { folha seca Colombo Drimys brasiliensis - } 120 \text { dias }\end{array}$ \\
\hline M4C2T4 & $\begin{array}{l}\text { Cachaça não envelhecida, com } 39,0 \% \text { de álcool com dois g L }{ }^{-1} \text { de } \\
\text { folha seca Colombo Drimys brasiliensis - } 120 \text { dias }\end{array}$ \\
\hline M4C3T4 & $\begin{array}{l}\text { Aguardente de cana não envelhecida, com } 39,0 \% \text { de álcool com dois } \\
\mathrm{g} \mathrm{L}^{-1} \text { de folha seca Colombo Drimys brasiliensis - } 120 \text { dias }\end{array}$ \\
\hline $\begin{array}{l}\text { C1 } \\
\text { C2 }\end{array}$ & $\begin{array}{l}\text { Cachaça não envelhecida, com } 47,5 \% \text { de álcool (Testemunha) } \\
\text { Cachaça não envelhecida, com 39,0\% de álcool (Testemunha) }\end{array}$ \\
\hline C3 & $\begin{array}{l}\text { Aguardente de cana não envelhecida, com } 39,0 \% \text { de álcool (Testemu- } \\
\text { nha) }\end{array}$ \\
\hline
\end{tabular}

A avaliação dos testes de preferência foi realizada a partir da expressão da opinião em uma Escala Hedônica. Estas foram usadas para avaliar o nível de preferência ou aceitação das amostras.

\section{Resultados e Discussões}

\section{Leitura de acidez $-1^{\circ}$ experimento}

Para as variáveis analisadas, tipos de folhas e tempos de infusão não houve interação entre os fatores analisados, demonstrando que estes são independentes. Houve diferença significativa ao nível de $1 \%$ de probabilidade para quantidade de folhas (Tabela 4). 
Tabela 4 - Comparação de médias das variáveis: quantidades de folhas de Drimys brasiliensis Miers e Pimenta pseudocaryophyllus (Gomes) Landrum: um g L ${ }^{-1}$ (Q1), dois $\mathrm{g} \mathrm{L}^{-1}(\mathrm{Q} 2)$ e quatro $\mathrm{g} \mathrm{L}^{-1}(\mathrm{Q} 3)$, com a infusão de folhas em cachaça não envelhecida com 39,0\% de álcool, para a leitura de acidez, Curitiba (PR), 2013.

$\begin{array}{cc}\text { Quantidade de Folhas } & \mathbf{g ~} \mathbf{1 0 0} \mathbf{~ m L} \\ \text { Q1 } & 0,15 \mathrm{c} \\ \text { Q2 } & 0,16 \mathrm{~b} \\ \text { Q3 } & 0,18 \mathrm{a}\end{array}$

Coeficiente de Variação (\%)

14,40

Médias seguidas de mesma letra na coluna (minúscula) não diferem estatisticamente ao nível de $5 \%$ de significância pelo Teste de Tukey. A acidez da cachaça é influenciada pela quantidade de folhas na infusão da cachaça, explicando, portanto os resultados do presente experimento, não havendo necessidade de apresentar assim as tabelas de interação.

A quantidade de quatro $\mathrm{g} \mathrm{L}^{-1}$ foi a que apresentou maior acidez seguida por dois $\mathrm{g} \mathrm{L}^{-1}$, sendo a quantidade de um $\mathrm{g} \mathrm{L}^{-1}$ a de menor acidez (Tabela 4). De acordo com Dantas et al. (2007), o grau de acidez das cachaças constitui fator de qualidade, pois, durante sua produção, os ácidos reagem com os álcoois presentes e contribuem para a formação de ésteres. Estes ésteres são importantes para o aroma, mas a acidez em excesso promove sabor indesejado e agressivo, reduzindo a qualidade da bebida (JERONIMO et al., 2008).

\section{Leitura de acidez -20 experimento}

Para as variáveis analisadas, tipos de cachaça e tempo de infusão, houve interação entre os fatores analisados, demonstrando 
que estes são dependentes. Houve diferença significativa ao nível de $1 \%$ de probabilidade para tipos de cachaça (Tabela 5). Para as cachaças não envelhecidas com 47,5\% e 39,0\% de álcool e aguardente de cana com 39,0\% de álcool, não houve diferença significativa quando relacionadas com o tempo de infusão (Tabela 5).

Ao analisar os tipos de cachaça, nota-se que para os tempos um, dois, três e quatro (trinta, sessenta, noventa e cento e vinte dias, respectivamente), a aguardente de cana não envelhecida com $39,0 \%$ de álcool (C3) foi a que apresentou menor acidez. Já a cachaça não envelhecida com 47,5\% de álcool (C1) e 39,0\% de álcool (C2) foram as mais ácidas em todos os tempos analisados, não havendo diferença significativa entre si (Tabela 5). E segundo Silva et al. (2010), a presença de ácidos nas bebidas alcóolicas contribuem significativamente no seu sabor.

Tabela 5 - Comparação de médias das variáveis tipos de cachaça (C1, C2 e C3) e tempo de infusão (T1, T2, T3 e T4) com a infusão de dois $\mathrm{g} \mathrm{L}^{-1}$ de folhas secas de Drimys brasiliensis Miers, para a leitura de acidez, Curitiba (PR), 2013.

\begin{tabular}{|c|c|c|c|c|}
\hline \multirow{3}{*}{ Tipos Cachaças } & \multicolumn{4}{|c|}{ Tempo de Infusão } \\
\hline & T1 & T2 & T3 & T4 \\
\hline & \multicolumn{4}{|c|}{ Acidez g $100 \mathrm{~mL}^{-1}$} \\
\hline C1 & $0,19 a A$ & $0,20 a A$ & $0,20 a A$ & $0,21 a A$ \\
\hline $\mathbf{C 2}$ & $0,16 \mathrm{aA}$ & $0,17 \mathrm{aA}$ & $0,16 a A$ & $0,18 \mathrm{aA}$ \\
\hline C3 & $0,08 \mathrm{bA}$ & $0,09 \mathrm{bA}$ & $0,09 \mathrm{bA}$ & $0,09 \mathrm{bA}$ \\
\hline Coeficiente de Variação (\%) & & & ,20 & \\
\hline \multicolumn{5}{|c|}{$\begin{array}{l}\text { Médias seguidas de mesma letra na coluna (minúscula) e na linha (maiúscula) não diferem estatisticamente ao } \\
\text { nível de } 5 \% \text { de significância pelo Teste de Tukey. }\end{array}$} \\
\hline \multicolumn{5}{|c|}{ C1T1: Cachaça não envelhecida com 47,5\% de álcool em trinta dias de infusão } \\
\hline \multicolumn{5}{|c|}{ C1T2: Cachaça não envelhecida com $47,5 \%$ de álcool em sessenta dias de infusão } \\
\hline \multicolumn{5}{|c|}{ C1T3: Cachaça não envelhecida com 47,5\% de álcool em noventa dias de infusão } \\
\hline \multicolumn{5}{|c|}{ C1T4: Cachaça não envelhecida com $47,5 \%$ de álcool em cento e vinte dias de infusão } \\
\hline \multicolumn{5}{|c|}{ C2T1: Cachaça não envelhecida com $39,0 \%$ de álcool em trinta dias de infusão } \\
\hline \multicolumn{5}{|c|}{ C2T2 Cachaça não envelhecida com $39,0 \%$ de álcool em sessenta dias de infusão } \\
\hline \multicolumn{5}{|c|}{ C2T3: Cachaça não envelhecida com $39,0 \%$ de álcool em noventa dias de infusão } \\
\hline \multicolumn{5}{|c|}{ C2T4: Cachaça não envelhecida com $39,0 \%$ de álcool em cento e vinte dias de infusão } \\
\hline \multicolumn{5}{|c|}{ C3T1: Aguardente de cana não envelhecida com 39,0\% de álcool em trinta dias de infusão } \\
\hline \multicolumn{5}{|c|}{ C3T2: Aguardente de cana não envelhecida com $39,0 \%$ de álcool em sessenta dias de infusão } \\
\hline \multicolumn{5}{|c|}{ С3Т3: Aguardente de cana não envelhecida com $39,0 \%$ de álcool em noventa dias de infusão } \\
\hline & & & & \\
\hline
\end{tabular}




\section{Leitura de cor $-1^{\circ}$ experimento}

Para as variáveis analisadas, tipos de folhas e quantidades de folhas houve interação entre os fatores analisados, demonstrando que estes são independentes. Houve diferença significativa ao nível de $1 \%$ de probabilidade para os tipos de folhas e quantidade de folhas (Tabela 6).

A quantidade de quatro $\mathrm{g} \mathrm{L}^{-1}$ de folhas secas de Pimenta peudocaryophyllus e de folhas secas e verdes de Drimys brasiliensis foram as que mostraram maior coloração das cachaças quando comparada com as quantidades de dois $\mathrm{g} \mathrm{L}^{-1}$ e $1 \mathrm{~g} \mathrm{~L}^{-1}$, sendo esta última a que menos corou a cachaça (Tabela 6). Isto está relacionada à maior quantidade de folhas em contato com a bebida o que provoca o aumento na coloração da mesma.

Tabela 6 - Comparação de médias das variáveis: tipos de folhas (M1, M2 e M3) e quantidade de folhas (Q1, Q2, e Q3) com a infusão de folhas secas de Pimenta pseudocaryophyllus (Gomes) Landrum e folhas secas e verdes de Drimys brasiliensis Miers em cachaça não envelhecida com 39,0\% de álcool, para a leitura de cor aos cento e vinte dias, Curitiba (PR), 2013.

\begin{tabular}{cccc}
\hline \multirow{2}{*}{ Tipos de Folhas } & \multicolumn{3}{c}{ Quantidade de Folhas } \\
\cline { 2 - 4 } M1 & $\mathrm{Q} 1$ & $\mathrm{Q} 2$ & $\mathrm{Q} 3$ \\
\hline M2 & $0,1 \mathrm{aC}$ & $0,4 \mathrm{aB}$ & $0,8 \mathrm{aA}$ \\
M3 & $0,0 \mathrm{bC}$ & $0,0 \mathrm{bB}$ & $0,2 \mathrm{bA}$ \\
Coeficiente de Variação $(\%)$ & $0,0 \mathrm{bC}$ & $0,0 \mathrm{bB}$ & $0,2 \mathrm{bA}$ \\
\hline
\end{tabular}

Médias seguidas de mesma letra na coluna (minúscula) e na linha (maiúscula) não diferem estatisticamente ao nível de $5 \%$ de significância pelo Teste de Tukey.

A variação de cor durante o tempo de infusão apresenta comportamento crescente, ou seja, quanto maior o tempo de infusão das folhas na cachaça, mais corada esta ficará. Assim, foi apresentada apenas à tabela de leitura de cor aos cento e vinte dias, pois foi a que apresentou maior tonalidade quando comparada com as demais leituras. Isto está relacionado à maior quantidade de folhas em con- 
tato com a bebida, o que provoca o aumento na coloração da mesma. E, o fato de haver uma maior coloração utilizando-se folhas de Pimenta pseudocayophyllus em relação às folhas de Drimys brasiliensis pode estar relacionado ao ambiente de cultivo, porém é preciso mais estudos para poder afirmar.

Durante o envelhecimento, a cachaça passa por inúmeras transformações, dentre elas a incorporação de componentes solúveis extraídos da folha e a decomposição parcial de macromoléculas em monômeros solúveis, como aldeídos e ácidos fenólicos, que são incorporados à cachaça. Todas estas transformações provocam o aumento progressivo da coloração e viscosidade (MAIA; CAMPELO, 2005). E de acordo com Méndelez-Martínez et al. (2007), para a maioria dos consumidores a cor é fator significativo, determinante de aceitação dos mesmos.

\section{Leitura de cor $-1^{\circ}$ experimento}

Para as variáveis analisadas, tipo de cachaça e tempo de infusão não houve interação entre os fatores, demostrando que estes são independentes. Para a variável tipo de cachaça houve diferença significativa ao nível de $5 \%$ de probabilidade (Tabela 7).

Tabela 7- Comparação de médias das variáveis: tipos de cachaça (C1, C2 e C3) com a infusão de folhas secas de Drimys brasiliensis Miers, para a leitura de cor aos cento e vinte dias, Curitiba (PR), 2013.

\begin{tabular}{cc}
\hline Tipos de Cachaça & $\mathbf{N m}$ \\
\hline C1 & $0,15 \mathrm{a}$ \\
C2 & $0,10 \mathrm{~b}$ \\
C3 & $0,13 \mathrm{ab}$ \\
\hline
\end{tabular}

\section{Coeficiente de Variação(\%)} 23,40

Médias seguidas de mesma letra na coluna (minúscula) não diferem estatisticamente ao nível de $5 \%$ de significância pelo Teste de Tukey. 
Houve um aumento crescente na cor da bebida conforme aumentavam os dias da leitura, mostrando que a leitura aos cento e vinte dias apresentou maior coloração na cachaça, por isso não houve a necessidade de apresentar as demais tabelas de leitura.

A cachaça não envelhecida com $47,5 \%$ de álcool foi a que apresentou maior coloração diferindo estatisticamente apenas da cachaça não envelhecida com 39,0\% de álcool (Tabela 7).

\section{Análise sensorial $-1^{\circ}$ experimento}

$\mathrm{Na}$ análise sensorial realizada, as amostras com a infusão de folhas de Pimenta pseudocaryophyllus (M1Q1T4, M1Q2T4 e M1Q3T4) não diferiram significativamente entre si bem como as amostras com a infusão de folhas de Drimys brasiliensis (M2Q1T4, M2Q2T4 e M2Q3T4) que também não diferiram estatisticamente entre si (Tabela 8).

Tabela 8 - Comparação de médias das variáveis de seis amostras de cachaça não envelhecida com a infusão de folhas secas de Pimenta pseudocaryophyllus (Gomes) Landrum com um $\mathrm{g} \mathrm{L}^{-1}$ (M1Q1T4), folhas secas de Pimenta pseudocaryophyllus (Gomes) Landrum com dois $\mathrm{g} \mathrm{L}^{-1}$ (M1Q2T4), folhas secas de Pimenta pseudocaryophyllus (Gomes) Landrum com quatro $\mathrm{g} \mathrm{L}^{-1}$ (M1Q3T4), folhas secas de Drimys brasiliensis Miers com um g L ${ }^{-1}$ (M2Q1T4), folhas secas de Drimys brasiliensis Miers com dois $\mathrm{g} \mathrm{L}^{-1}$ (M2Q2T4), folhas secas de Drimys brasiliensis Miers com quatro $\mathrm{g} \mathrm{L}^{-1}$ (M2Q3T4), Curitiba (PR), 2013.

\begin{tabular}{cc}
\hline Amostras & Nota \\
\hline M1Q1T4 & $4,6 \mathrm{ab}$ \\
M1Q2T4 & $4,9 \mathrm{ab}$ \\
M1Q3T4 & $5,0 \mathrm{a}$ \\
M2Q1T4 & $4,2 \mathrm{bc}$ \\
M2Q2T4 & $4,3 \mathrm{bc}$ \\
M2Q3T4 & $3,9 \mathrm{c}$ \\
\hline Coeficiente de Variação (\%) & 29,3 \\
\hline
\end{tabular}

Médias seguidas de mesma letra na coluna (minúscula) não diferem estatisticamente ao nível de $5 \%$ de significância pelo Teste de Tukey. 
A cachaça com o uso de quatro $\mathrm{g} \mathrm{L}^{-1}$ de folhas de Pimenta pseudocaryophyllus foi a que apresentou melhor aceitação pelo consumidor quando comparada com as demais amostras julgadas (Tabela 8). E a cachaça com a infusão de folhas secas de Drimys brasiliensis na mesma quantidade de quatro $\mathrm{g} \mathrm{L}^{-1}$, foi a única que se diferenciou estatisticamente da cachaça com a infusão de folhas secas de Pimenta pseudocaryophyllus, sendo ainda a cachaça que o consumidor menos gostou quando comparada com as demais.

\section{Análise sensorial $-2^{\circ}$ experimento}

Pela análise de variância os dados da composição centesimal da análise sensorial exibem que não houve diferença significativa entre si, mostrando que todas foram igualmente aceitas pelo consumidor (Tabela 9). Odello et al. (2009), afirma que conhecer o nível de preferência do produto é importante, porém saber quais os caracteres que afetam positiva ou negativamente um produto é o ponto principal a fim de poder-se melhorar as suas técnicas de processamento.

Tabela 9 - Comparação de médias das variáveis de seis amostras com a infusão de dois $\mathrm{g} \mathrm{L}^{-1}$ de folhas secas de Drimys brasiliensis Miers: cachaça não envelhecida com $47,5 \%$ de álcool (M4C1T4), cachaça não envelhecida com 39,0\% de álcool (M4C2T4), aguardente de cana não envelhecida com 39,0\% de álcool (M4C3T4), cachaça não envelhecida com $47,5 \%$ de álcool sem a infusão de folhas (C1), cachaça não envelhecida com 39,0\% de álcool sem a infusão de folhas (C2), aguardente de cana não envelhecida com $39,0 \%$ de álcool sem a infusão de folhas (C3), Curitiba (PR), 2013.

\begin{tabular}{cc}
\hline Amostras & Nota \\
\hline M4C1T4 & $4,1 \mathrm{a}$ \\
M4C2T4 & $4,1 \mathrm{a}$ \\
M4C3T4 & $4,4 \mathrm{a}$ \\
C1 & $4,1 \mathrm{a}$ \\
C2 & $4,4 \mathrm{a}$ \\
C3 & $3,8 \mathrm{a}$ \\
\hline Coeficiente de Variação (\%) & 33,3 \\
\hline
\end{tabular}

Médias seguidas de mesma letra na coluna (minúscula) não diferem estatisticamente ao nível de $5 \%$ de significância pelo Teste de Tukey. 


\section{Conclusão}

A cachaça com quatro $\mathrm{g} \mathrm{L}^{-1}$ de folhas secas de Pimenta pseudocaryophyllus (Gomes) Landrum e folhas secas e verdes de Drimys brasiliensis Miers e a cachaça não envelhecida com $47,5 \% \mathrm{e}$ $39,0 \%$ de álcool com a infusão de dois $\mathrm{g} \mathrm{L}^{-1}$ de folhas secas de Drimys brasiliensis Miers apresentaram maior acidez. Quanto maior o tempo de infusão de quatro $\mathrm{g} \mathrm{L}^{-1}$ de folhas secas e verdes de Drimys brasiliensis Miers e folhas secas de Pimenta pseudocaryophyllus (Gomes) Landrum na cachaça, maior a sua coloração.

A cachaça com quatro $\mathrm{g} \mathrm{L}^{-1}$ de folhas secas de Pimenta pseudocaryophyllus (Gomes) Landrum foi a melhor aceita pelo consumidor em comparação com a cachaça com folhas secas de Drimys brasiliensis. As cachaças com a infusão de dois $\mathrm{g} \mathrm{L}^{-1}$ de folhas secas de Drimys brasiliensis Miers em diferentes teores alcoólicos de cachaça não envelhecida e aguardente de cana foram igualmente aceitas pelo consumidor.

\section{Referências}

BRASIL. Portaria oㅜ 76, de 26 de novembro de 1986. Diário oficial [da] República Federativa do Brasil, 28 nov. 1986.

DANTAS, H. J. et al. Avaliação da influência da velocidade de destilação na análise físico-química de aguardente de cana de açúcar. Revista Brasileira de Produtos Agroindustriais, v. 9, n. 2, p. 101109, 2007. 
GIRARD, E. A. Volume, biomassa e rendimento de óleos essências do craveiro (Pimenta pseudocaryophyllus (Gomes) Landrum) em Floresta Ombrófila mista. 2005. 72 f. Dissertação (Mestrado em Manejo Florestal) - Programa de Pós-Graduação em Engenharia Florestal, Universidade Federal do Paraná, Curitiba. 2005.

JERONIMO, E. M. et al. Addition of proteic nitrogen during fermentation for the production of cachaça. Scienc Agricultural, v. 65, n. 2, p. 161-168, 2008.

MAIA, A. B. R. A.; CAMPELO, E. A. P. Tecnologia da cachaça de Alambique. Minas Gerais: SEBRAE/SINBEBIDAS, 2005. 129 p.

MARINHO, A. V.; RODRIGUES, J. P. M.; SIQUEIRA, M. I. D. Avaliação da acidez volátil, teor alcóolico e de cobre em cachaças artesanais. Revista Estudos, v. 36, n. 1/2, p. 75-93, 2009.

MÉNDELEZ-MARTíNEZ, A. J. et al. Relationship between the colour and the chemical structure of carotenoid pigments. Food Chemical, v. 101, p. 1145-1150, 2007.

MIRANDA, M. B. et al. Qualidade química de cachaças e de aguardentes brasileiras. Ciência e Tecnologia de Alimentos, v. 27, n. 4, p. 897-901, 2007.

MIRANDA, M. B. et al. Perfil físico-químico de aguardente durante envelhecimento em tonéis de carvalho. Ciência e Tecnologia de Alimentos, v. 28, p. 84-89, 2008. 
ODELLO, L. et al. Avaliação sensorial de cachaça. Química Nova, v. 32, n. 7, p. 1839-1844, 2009.

PARAZZI, C. et al. Avaliação e caracterização dos principais compostos químicos da aguardente de cana-de-açucar envelhecida em tonéis de carvalho (Quercus sp.). Ciência e Tecnologia de Alimentos, v. 28, n. 1, p. 193-199, 2008.

RECHE, R. V.; FRANCO, D. W. Distinção entre cachaças destiladas em alambiques e em colunas usando quimiometria. Química Nova, v. 32, n. 2, p. 332-336, 2009.

SAKAI, R. H. Árvore do conhecimento da cana de açúcar. Brasília, 2010.

Disponível em:

<http://www.agencia.cnptia.embrapa.br/gestor/cana-deacucar/arvore/CONT000fiog1ob502wyiv80z4s473agi63ul.html>. Acesso em: 10 jan. 2017.

SILVA, J. L. A. et al. Utilização de abacaxi para a elaboração de vinhos: avaliação físico química e aceitabilidade. Holos, v. 26, n. 3, p. 108-118, 2010.

SORATTO, A. N.; VARVAKIS, G.; HORII, J. A certificação agregando valor à cachaça do Brasil. Ciência e Tecnologia de Alimentos, v. 27, n. 4 , p. 681-687, 2007.

TRINTA, E. F; SANTOS, E. Flora Ilustrada Catarinense. Itajaí: BR Petrobras, 1997. 
ZEM, L. M. Drymis brasiliensis: propagação vegetativa, composição química do óleo essencial e infusão alcóolica de folhas. 2014. 189 f. Dissertação (Mestrado Agronomia) - Programa de PósGraduação em Agronomia, Departamento de Fitotecnia e Fitossanitarismo, Universidade Federal do Paraná, 2014. Disponível em: $<$ http://acervodigital.ufpr.br/bitstream/handle/1884/35345/R\%20\%20D\%20-\%20LUCIELE\%20MILANI\%20ZEM.pdf?sequence=1 >. Acesso em: 18 jan. 2017. 